\title{
Decoding and Functional Analysis of Genetic Programs of Hybrid Electromechanical Structures
}

\author{
Vasyl Shynkarenko ${ }^{1}$, Iurii Gaidaienko ${ }^{1} \&$ Ahmad N. Al-Husban ${ }^{2}$ \\ ${ }^{1}$ National Technical University of Ukraine 'Kyiv Polytechnic Institute', Ukraine \\ ${ }^{2}$ Faculty of Engineering Technology, Al-Balqa Applied University, Amman, Jordan \\ Correspondence: Ahmad N. Al-Husban, Faculty of Engineering Technology, Al-Balqa Applied University, \\ Amman 11974, Jordan. Tel: 962-777-432-822. E-mail: drahusban2008@Yahoo.com
}

\author{
Received: January 3, $2014 \quad$ Accepted: February 3, $2014 \quad$ Online Published: February 17, 2014 \\ doi:10.5539/mas.v8n2p36 URL: http://dx.doi.org/10.5539/mas.v8n2p36
}

\begin{abstract}
The results of decoding and genetic programs of structuration analysis of hybrid electromechanical systems are generalized in this paper. Authors established the determined interrelations between genetic programs, structure of periods and groups in periodic generating system of electromagnetic field primary sources, genetic codes of hybrid chromosomes, the taxonomical and functional variety of developing electromechanical objects of hybrid type. The quantitative structure and properties of genetically admissible variety of hybrid Types of electromechanical objects are defined. The results of evolutionary experiments confirming reliability of predictive function of genetic programs are given in this article.
\end{abstract}

Keywords: electromechanical structure, electromagnetic chromosome, genetic code, genetic program, topology, electromagnetic symmetry, hybrid structure, evolutionary experiment

\section{Introduction}

The global changes of the world around happening recently in the nature, society and techno sphere put forward essentially new tasks for modern science. Evolutionarily the mankind came closely to understanding of the thing that society has to become part of the nature, to live due to the essentially new, inexhaustible resources and technologies, modeled on the wildlife similitude, but with use of the most perfect technological achievements (Kovalchuk, 2011; Wang et al., 2000). Practical realization of this way goes through the understanding and utilization of system laws of the nature. The evolution and genetics laws, coming out in a specific way in systems of both natural and an anthropogenic origin (Shinkarenko, 2013; Tadmor, 2002), are among the all-system laws.

The electromechanical energy converters belong to the developing of a natural-and-anthropogenic system class, which structural organization and development are defined by the all-system laws of evolution and the principles of heredity (Shinkarenko, 2002; Breedveld, 2001). One of the dominating tendencies in the equipment evolution is directly connected with creation and wide application of hybrid objects and systems. The genetic principle of hybridization belongs to one of the most productive mechanisms in complex systems development, extending not only on biological, but also anthropogenic systems (Venda, 1990; Kalachek, Koriagin, Kolesnikov, \& Minkova, 2011; Kuznietsov \& Dmitriev, 2009). The understanding of the structural organization principles of hybrid objects is a key to knowledge harmonization and understanding of operated evolution patterns of complex systems.

The theoretical bases were developed (Shinkarenko, 2002; Shinkarenko \& Gaidaienko, 2010a, 2010b; Shinkarenko, Gaidaienko, \& Ahmad, 2013) and the fundamental principles of the hybrid electromechanical objects (EM-objects) structural organization were scientifically proved by the previous researches, which results can be generalized by the following ideas:

- Hybrid EM-objects have the genetic nature since their synthesis is based on the all-system principle of crossing of the initial (parental) electromagnetic chromosomes, differing by their genetic information (Shinkarenko, 2002; Shinkarenko \& Gaidaienko, 2010a);

- The carriers of hereditary genetic information of hybrid objects are universal genetic codes of the crossed parental chromosomes which set is ordered by the genetic classification (GC) of primary sources of the 
electromagnetic field (Shinkarenko, 2002; Rodriguez \& Ortega, 2003);

- The mechanism of the genetic information transfer between generations of the developing anthropogenic systems, including EM-objects, is carried out through the personal intelligence in the form of scientific publications, patents, design documentation, etc. (Shinkarenko, 2013; Chiasson, 2005);

- Genetically admissible variety of functional classes of hybrid electromechanical structures (EM-structures) is predetermined by their genetic programs, which contain generating sets of electromagnetic chromosomes and have certain predictive function (Shinkarenko, 2013; Shinkarenko et al., 2013);

- The potentially possible variety of hybrid objects is divided by quantity of crossed components of genetic information into three classes: mono- , di- and poly-hybrids (Shinkarenko \& Gaidaienko, 2010a);

- Hybrid objects have emergent properties, i.e., as a result of crossing they gain new properties and functions which the initial structures do not have (Shinkarenko \& Gaidaienko, 2010a, 2010b);

- Theoretical basis for the analysis and synthesis of hybrid EM-structures is formed by the genetic models of convergent type and methods of genetic combination theory (Shinkarenko \& Gaidaienko, 2010a);

- Reliability of theoretical provisions of EM-objects' genetic evolution is confirmed by conducted evolutionary experiments (Shinkarenko \& Kotlyarova, 2012; Shinkarenko, Shvedchikova, \& Kotlyarova, 2013; Shvedchikova, 2013).

The new scientific results received recently in the field of genetic electro mechanics, radically change approach to the theoretical analysis and structural synthesis of complex EM-objects. The system approach basis in the research of developing classes of hybrid objects is formed by the genetic programs containing ordered highly intellectual information about known structural representatives as well as about genetically admissible objects, but still absent on this time of their evolution. Definition and decoding of genetic structuration programs of EM-objects belongs to a prerogative of new developing field of knowledge, namely Predictive Genomics of Anthropogenic Systems, and researches in this direction represent an actual scientific task.

\subsection{Problem Formulation}

This paper considers the problem of decoding of genetic programs by criterion of genetic information components and the definition of determined interrelation between programs and steady hereditary features (electromagnetic, topological and geometrical) of hybrid classes of descendant EM-structures. Fundamental nature of genetic information is defined by its invariance to the complexity level, functional belonging and time of evolution of descendant objects. Decoding and analysis of genetic programs allows to define not only quantitative composition of generating structures and Species of hybrid EM-objects, but also to predict hereditary properties of descendant structures.

Let us consider the analysis problem at the element level presented by primary sources of electromagnetic field, which set is ordered in the domain of GC. In terms of the genetic theory the concept of primary source of a field is equivalent to a concept of parental electromagnetic chromosome. In order to ensure a correctness of the solution to the problem, we will accept the following assumptions:

- The initial set of parental chromosomes is limited by the element basis of the first big period of genetic classification;

- In this research the twin hybrids, synthesized on the basis of isotope sources of field aren't considered;

- We limit level of genetic complexity of descendant structures by the first generation of hybrids (i.e., we don't consider isomer groups and complex hybrid compositions);

- The intraspecific hybrid structures containing secondary sources of a magnetic field are not considered.

Level of generalization of genetic models and genetic programs considered below is defined under the assumptions made.

\section{Principles of Structurization}

The principles of structural organization of hybrid systems are in details investigated and published in the previous works of authors (Shinkarenko, 2002; Shinkarenko \& Gaidaienko, 2010a, 2010b; Shinkarenko et al., 2013). In this article the specified principles are analyzed by criterion of functional properties of hybrid EM-structures.

The fundamental for hybrid EM-structures synthesis is the all-system principle of crossing of genetically different parental electromagnetic chromosomes (Shinkarenko \& Gaidaienko, 2010a; Chiasson, 2005) 


$$
A \times B \rightarrow H_{i j}
$$

Where chromosomes $A$ and $B$ are presented by their genetic codes: $A=\left(a_{1}, a_{2}, a_{3}\right) ; B=\left(b_{1}, b_{2}, b_{3}\right) ; a_{1}, a_{2}, \ldots, b_{1}$, $b_{2}, \ldots$ - are the components of genetic information.

The universal genetic code of any electromagnetic chromosome contains three components of genetic information which code spatial (geometrical), electromagnetic and topological properties of descendant structures, define the main taxonomical signs and location (coordinates) of a parental chromosome in periodic structure of GC (Figure 1).

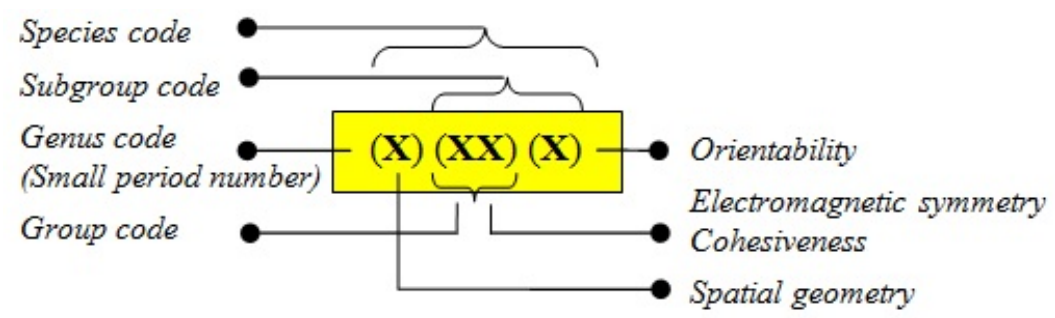

Figure 1. Interrelations of genetic information components in structure of a universal genetic code of primary source of electromagnetic field (parental electromagnetic chromosome)

It is possible to separate the following classes of hybrid descendant structures by criterion of crossed components of genetic information:

- $\quad$ Spatial monohybrids $\left(a_{1} \neq b_{1} ; a_{2}=b_{2}=0 ; a_{3}=b_{3}=0\right)$ :

$$
H_{G}=\left(a_{1}\right) \times\left(b_{1}\right)
$$

- $\quad$ Electromagnetic monohybrids $\left(a_{2} \neq b_{2} ; a_{1}=b_{1}=0 ; a_{3}=b_{3}=0\right)$ :

$$
H_{E}=\left(a_{2}\right) \times\left(b_{2}\right)
$$

- $\quad$ Topological monohybrids $\left(a_{3} \neq b_{3} ; a_{1}=b_{1}=0 ; a_{2}=b_{2}=0\right)$ :

$$
H_{T}=\left(a_{3}\right) \times\left(b_{3}\right)
$$

In the case of dihybrid crossing (Figure 3 ) three classes of hybrids of the mixed type are formed:

- $\quad$ The spatially-electromagnetic $\left(a_{1} \neq b_{1} ; a_{2} \neq b_{2} ; a_{3}=b_{3}=0\right)$ :

$$
H_{G E}=\left(a_{1}, a_{2}\right) \times\left(b_{1}, b_{2}\right)
$$

- $\quad$ The electromagnetic and topological $\left(a_{2} \neq b_{2} ; a_{3} \neq b_{3} ; a_{1}=b_{1}=0\right)$ :

$$
H_{T E}=\left(a_{2}, a_{3}\right) \times\left(b_{2}, b_{3}\right)
$$

- $\quad$ The spatially-topological $\left(a_{1} \neq b_{1} ; a_{3} \neq b_{3} ; a_{2}=b_{2}=0\right)$ :

$$
H_{G T}=\left(a_{1}, a_{3}\right) \times\left(b_{1}, b_{3}\right)
$$

As a result of polyhybrid crossing $\left(a_{1} \neq b_{1} ; a_{2} \neq b_{2} ; a_{3} \neq b_{3}\right)$ one class of the complex hybrids combining differences in all of three properties is formed:

$$
H_{G T E}=\left(a_{1}, a_{2}, a_{3}\right) \times\left(b_{1}, b_{2}, b_{3}\right)
$$

The considered creation principles of hybrid structures, Equations $(2-8)$, are generalized by a diagonal matrix (Table 1) which displays the space of genetically admissible crossings according to the components of genetic information. Each hybrid chromosome carries out function of generating unit in relation to the corresponding set of descendant Species which multiplicity will be defined by genetic predisposition of crossed chromosomes, their generic assignment and the accepted assumptions. 
Table 1. Matrix of space of admissible crossings on the basis of inherited genetic information

\begin{tabular}{ccccccccc}
\hline \multirow{2}{*}{$\begin{array}{c}\text { Components of genetic } \\
\text { information of } \\
\text { chromosome } \boldsymbol{B}\end{array}$} & \multicolumn{6}{c}{ Components of genetic information of chromosome $\boldsymbol{A}$} \\
\cline { 2 - 9 } & $b_{1}$ & $a_{1}$ & $a_{2}$ & $a_{3}$ & $a_{1}, a_{2}$ & $a_{2}, a_{3}$ & $a_{1}, a_{3}$ & $a_{1}, a_{2}, a_{3}$ \\
\hline \multirow{3}{*}{$m=1$} & $b_{2}$ & 0 & $\boldsymbol{E}$ & 0 & 0 & 0 & 0 & 0 \\
& $b_{3}$ & 0 & 0 & $\boldsymbol{T}$ & 0 & 0 & 0 & 0 \\
\hline \multirow{3}{*}{$m=2$} & $b_{1}, b_{2}$ & 0 & 0 & 0 & $\boldsymbol{G E}$ & 0 & 0 & 0 \\
& $b_{2}, b_{3}$ & 0 & 0 & 0 & 0 & $\boldsymbol{T E}$ & 0 & 0 \\
\hline$m=3$ & $b_{1}, b_{3}$ & 0 & 0 & 0 & 0 & 0 & $\boldsymbol{G T}$ & 0 \\
\hline
\end{tabular}

The matrix structure can be considered as a way of presentation of the genetic program of hybrid EM-objects. The program shows interrelation between quantity of crossed signs $(m)$, components of genetic information $\left(a_{i}\right.$, $\left.b_{j}\right)$, quantity of generating functional chromosomes of hybrid type $(G, E, T, \ldots)$ and their emergent hereditary properties (spatial geometry, electromagnetic symmetry and topology of crossed active surfaces) which will define hereditary characteristics of hybrid descendant structures.

\section{Levels of Representation of Knowledge}

Hybridization principles are performed at all levels of genetic complexity of developing systems. Levels of genetic complexity of hybrid EM-objects are defined by the determined sequence: "Periodic structure of GC" $\rightarrow$ "Parental chromosome" $\rightarrow$ "Hybrid pair chromosome" $\rightarrow$ "Genetic program of Species" $\rightarrow$ "Species of hybrid EM-objects" $\rightarrow$ "Genetic program of a functional class" $\rightarrow$ "The functional class of hybrid EM-objects".

Proceeding from the specified relationships, it is possible to separate 6 structural organization levels of hybrid systems forming hierarchical sequence by criterion of genetic complexity (Figure 2). The hierarchy of levels forms hereditary structure. Each higher level comprises information of the lower one. Each level corresponds to a certain class of problems, genetic models and an appropriate level of structural and functional complexity of objects.

Initial level is chromosomal, presented by genetic programs of hybrid EM-objects

$$
P_{G}=\left(H_{G}, H_{E}, H_{T}, H_{G E}, H_{G T}, H_{T E}, H_{G T E}\right)
$$

Realization of crossing procedures and interpretation of genetic programs can be realized only having the genetic codes. This is the initial level of complexity in the structural organization of hybrid structures and it is presented in Figure 2 as chromosomal level. Problems of identification of genetic codes and genetic synthesis operators, problems of hybridization principles definition, procedures of definition and decoding of genetic programs, and also problems of classification and genetic systematization of hybrid classes of EM-objects belong to the main objectives of chromosomal level.

Specific level is presented by problems of structural synthesis and structural optimization of hybrid structures: spatial $\left(a_{11} \neq a_{12}\right)$, electromagnetic (distinctive symmetry of interacting fields) and topological (distinctive topology of active parts of primary and secondary sources of field). The principles of hybridization, the system analysis and classification of intraspecific hybrids represent an independent problem and are not considered in this paper. Problems of synthesis and structural optimization of hybrid objects belong to applied tasks and form a separate task of engineering level. Solving methods for such tasks are published by authors in the previous works, for example (Yerosh, 2001; Shinkarenko, Kuznietsov, Gaidaienko, \& Oliynyk, 2013). 


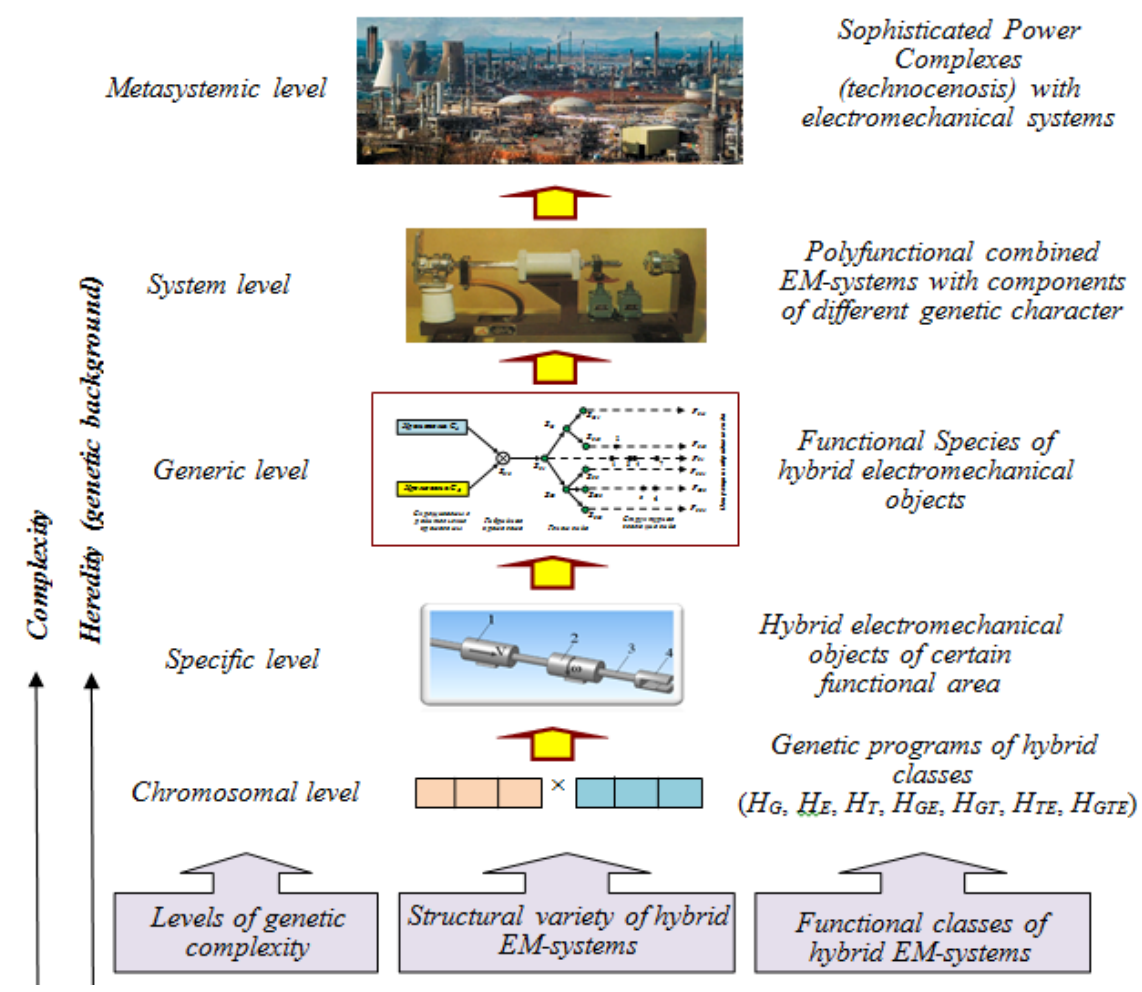

Figure 2. Levels of representation of knowledge in the problems of genetic analysis and synthesis of hybrid EM-structures

The third level of hierarchy (generic level) is presented by topological-and-electromagnetic mono- and dihybrid Species of EM-structures (including Species of twin hybrids) (Shinkarenko, Gaidaienko, \& Miroshnik, 2010) which objects are defined by the twoness principle. Such objects are formed by crossing of genetic codes of the chromosomes belonging to one geometrical class of EM-objects $\left(a_{1}=b_{1}=0\right)$. Synthesis of such hybrids is possible only within a concrete genus of EM-structures. Problems of synthesis and analysis of EM-hybrids at intrageneric level are in detail considered in the previous publications of authors (Shinkarenko et al., 2013; Shinkarenko \& Gaidaienko, 2010b). Problems of synthesis and analysis of polyhybrids which are forming only at intergeneric crossing, Equation (8), belong to problems of the third level of hierarchy also.

Classes of the electromechanical systems which combine components of subsystems of other genetic character (mechanical, thermal, hydraulic, the mechatronic, biological, etc.) belong to hybrid compositions of system level Emergent properties of such systems are defined by their specific polyfunctionality which is absent in initial subsystems. Compatibility is meant as such similarity of objects of various genetic character which provides possibility of their interaction and functioning.

Sophisticated, spatially distributed, integrated complexes (techno enosis) with electromechanical systems of energy generation and transformation form met systemic level of genetic, structural and functional complexity.

\section{Analysis of Genetic Programs}

The main objectives of genetic program analysis are problems of definition of quantitative structure and identification of admissible combinations genetic information components in generating classes of hybrid EM-structures. The quantitative structure of genetically admissible variety of hybrid structures at chromosomal level is determined by a known formula of a combination - selections (Yerosh, 2001).

$$
N_{n}{ }^{m}=n ! / m !(n-m) !
$$

Taking into accounting the accepted assumptions, for 6 geometrical classes of chromosomes $(n=6)$ of the first big period, genome $H_{G}$ of the class, according to Equation (10), within any subgroup will be defined by 15 spatial monohybrids:

$$
\left(N_{G}\right)_{6}^{2}=6 \cdot 5 \cdot 4 \cdot 3 \cdot 2 / 2(6-2) \cdot 3 \cdot 2=15
$$


The set of chromosomes $N_{G}$ corresponds to the following spatial combinations:

$$
\begin{gathered}
H_{G}=\left[G_{(C L \times K N)} ; G_{(C L \times P L)} ; G_{(C L \times T P)} ; G_{(C L \times S P)} ; G_{(C L \times T C)} ; G_{(K N \times P L)} ; G_{(K N \times T P)} ; G_{(K N \times S P)} ;\right. \\
\left.G_{(K N \times T C)} ; G_{(P L \times T P)} ; G_{(P L \times S P)} ; G_{(P L \times T C)} ; G_{(T P \times S P)} ; G_{(T P \times T C)} ; G_{(S P \times T C)}\right]
\end{gathered}
$$

The initial set of spatial chromosomes $H_{G}$ represents the genetic subprogram of admissible morphological compositions of hybrid type in the first generation. The element basis of the subprogram (12) forms a basis for definition and solving of system problems of analysis and synthesis of both primary, and secondary hybrid structures of spatial type. Specifically, the subprogram $H_{G}$ was successfully applied to definition of genetically admissible boundaries of structurization for electrical motors with rolling rotor (Shinkarenko, Naniy, Kotlyarova, $\&$ Dunev, 2012). As the periodic structure of GC includes 6 subgroups, then the genetically admissible variety of hybrid chromosomes $\sum H_{G}$ within the first big period will be defined by 90 spatial combinations.

The mono- and dihybrid crossing on a spatial characters is directly connected with formation of active surfaces (pole-forming structures, multiphase windings), combining sources with various spatial geometry. Synthesis of hybrid structures of spatial type is genetically admissible only on condition of crossing of homologous, topological equivalent chromosomes of intergeneric level. As the examples of such objects would be some kinds of electric motors with rolling rotor (intraspecific hybrids), the combined electromechanical converters of "screw-nut"-type, the combined EM-objects with toroid and cylindrical (Figure 3) and toroid-and-planar active surfaces (intergeneric dihybrids), the combined inductors for realization of plane-parallel motion, etc.

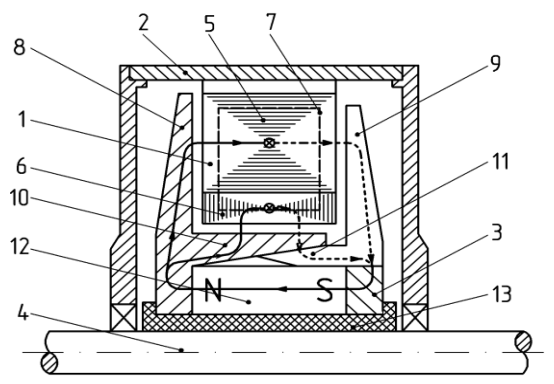

Figure 3. The contactless synchronous generator from toroid and cylindrical $(T C \times 2 T P)$ an active surface and hybrid system of poles of a rotor (the representative of a class of spatial hybrids of $H_{G}$ ) (Shinkarenko, Chumack, Gaidaienko, \& Vyshnevskyy, 2012)

The element basis of electromagnetic chromosomes $H_{E}$ in periodic structure of GC is defined by 4 groups $(n=4)$ of electromagnetic symmetry $(0.0 ; 0.2 ; 2.0$ and 2.2). Therefore, the quantitative composition of such monohybrid structures within any group according to (9) will be defined by 6 generating chromosomes -3 for each subgroup of the field sources: with $y$-oriented $(0.0 ; 0.2$ and 2.2$)$ and $x$-oriented waves of electromagnetic field $(0.0 ; 2.0$ and 2.2$)$

$$
\begin{aligned}
H_{E y} & =\left[E_{(00 \times 02) y} ; E_{(00 \times 22) y} ; E_{(02 \times 22) y}\right] \\
H_{E x} & =\left[E_{(00 \times 20) x} ; E_{(00 \times 22) x} ; E_{(20 \times 22) x}\right]
\end{aligned}
$$

Generating chromosomes in Equations $(13-14)$ and their descendants have active parts with the combined group of electromagnetic symmetry and topology. The total number of monohybrid chromosomes of the electromagnetic type $\sum N_{E}$ within the first big period of GC is 36 .

The specific feature of electromagnetic and topological-and-electromagnetic hybrids is existence of differences in electromagnetic symmetry of the combined active surfaces. Electrical machines with arc inductors (intraspecific hybrids) and also windings and inductors combining superficial and ring types of distributed windings (Figure 4) belong to such EM-objects. 


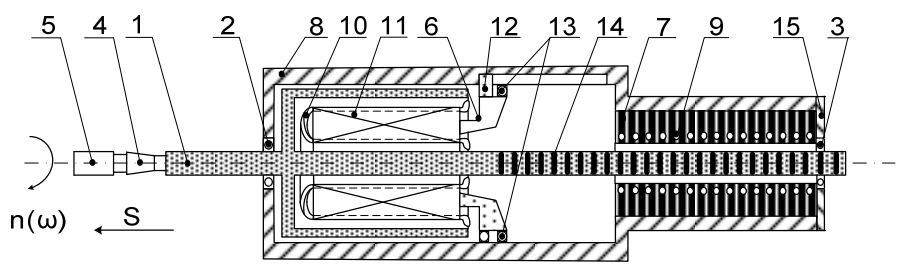

Figure 4. The combined electromechanical Motor spindle system (the representative of a class topological electromagnetic hybrids $H_{T E}$ ) (Shinkarenko, Kuznietsov, Gaidaienko, Kovtun, \& Oliynyk, 2013)

According to the principle of a dissimmetrization of P. Curie (Sonin, 2006), the integrated group of symmetry of hybrid descendant structures is defined by symmetry of the initial chromosome that has higher level of electromagnetic dissymmetry. As a result of electromagnetic symmetry disturbance at chromosomal level there will be corresponding types of primary electromagnetic end-effects at the level of descendant objects (Table 2). End effects can be considered as the demonstration of some kinds of "hereditary diseases", which are developing in operation of EM-objects.

Table 2. Interrelations between the electromagnetic dissymmetry of generating chromosomes and the type of electromagnetic end-effects in the hybrid classes $H_{E}$

\begin{tabular}{|c|c|c|c|c|c|c|}
\hline & \multicolumn{6}{|c|}{ Electromagnetic dissymmetry of the crossed chromosomes } \\
\hline & $(0.0 \times 0.2) y$ & $(0.0 \times 2.0) x$ & $(0.0 \times 2.2) y$ & $(0.0 \times 2.2) x$ & $(0.2 \times 2.2) y$ & $(2.0 \times 2.2) x$ \\
\hline & \multicolumn{6}{|c|}{ Resulting electromagnetic dissymmetry of hybrid chromosomes $H_{E}$} \\
\hline & 0.2 & 2.0 & 2.2 & 2.0 & 2.2 & 2.2 \\
\hline $\begin{array}{c}\text { Type of } \\
\text { the } \\
\text { end-effect }\end{array}$ & Transverse & Longitudinal & $\begin{array}{c}\text { Transverse-and- } \\
\text { longitudinal }\end{array}$ & $\begin{array}{c}\text { Transverse-and- } \\
\text { longitudinal }\end{array}$ & $\begin{array}{c}\text { Transverse-and- } \\
\text { longitudinal }\end{array}$ & $\begin{array}{c}\text { Transverse-and- } \\
\text { longitudinal }\end{array}$ \\
\hline
\end{tabular}

The quantitative and qualitative structure of generating chromosomes of the topological type $H_{T}$ is defined by orientability feature which is coded by the third component of genetic information in the genetic code structure (Figure 1). In the case of monohybrid crossing within any genus it is possible to create only two hybrid chromosomes of this type which descendants at specific level are generalized by the concept of twinned Species (Figure 5).
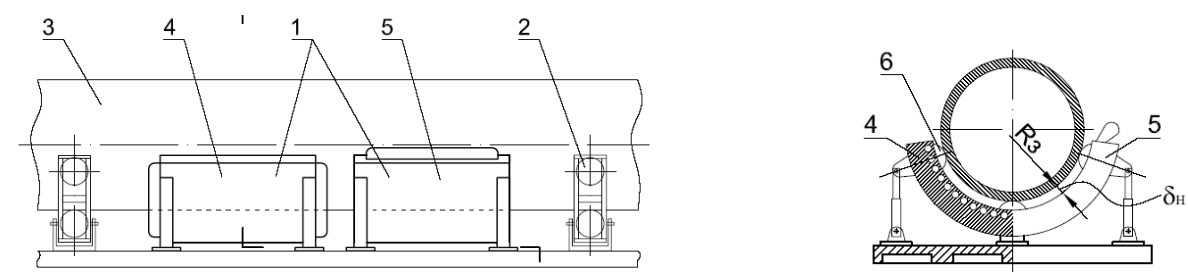

Figure 5. The combined electromechanical system for the steel pipes manipulator (the representative of a class of topological monohybrids of the twinned type $H_{T}$ ) (Shinkarenko, Chumack, Gaidaienko, \& Miroshnyk, 2010)

Within the first big period the quantity of topological chromosomes is: $N_{T}=2 \cdot 6=12$, and their genetic variety is presented by the field sources with orthogonally oriented magnetic fields

$$
\begin{array}{r}
\sum H_{T}=[C L O .0(x \times y) ; C L 2.2(x \times y) ; K N O .0(x \times y) ; K N 2.2(x \times y) ; P L O .0(x \times y) ; \\
P L 2.2(x \times y) ; T P O .0(x \times y) ; T P 2.2(x \times y) ; S P O .0(x \times y) ; S P 2.2(x \times y) ; \\
T C 0.0(x \times y) ; T C 2.2(x \times y)]
\end{array}
$$

The general property of hybrid EM-objects of topological type is the existence of orthogonal resultant fields (including combinations with running, rotating or spatially-concentric waves of a field). 
In case of dihybrid crossing (on the second and third component of genetic codes), the initial set of generating structures $H_{T E}$ can be defined using a matrix method (Table 3 ).

Table 3. Matrix of admissible crossings of dihybrid EM-structures of topological-and-electromagnetic type $\left(H_{T E}\right)$

\begin{tabular}{|c|c|c|c|c|c|c|}
\hline \multirow{2}{*}{$\begin{array}{c}\text { Components of } \\
\text { genetic information } \\
\text { of chromosome } \boldsymbol{B}\end{array}$} & \multicolumn{6}{|c|}{ Components of genetic information of chromosome $\boldsymbol{A}$} \\
\hline & $0.0 y$ & $0.0 x$ & $0.2 y$ & $2.0 x$ & $2.2 y$ & $2.2 x$ \\
\hline $0.0 y$ & - & - & - & $(0.0 y \times 2.0 x)$ & - & $(0.0 y \times 2.2 x)$ \\
\hline $0.0 x$ & - & - & $(0.0 x \times 0.2 y)$ & - & $(0.0 x \times 2.2 y)$ & - \\
\hline $0.2 y$ & - & $(0.0 x \times 0.2 y$ & - & $(0.2 y \times 2.0 x)$ & - & $(0.2 y \times 2.2 x)$ \\
\hline $2.0 x$ & $(0.0 y \times 2.0 x$ & - & $(0.2 y \times 2.0 x)$ & - & $(2.0 x \times 2.2 y)$ & - \\
\hline $2.2 y$ & - & $(0.0 x \times 2.2 y$ & - & $(2.0 x \times 2.2 y)$ & - & - \\
\hline $2.2 x$ & $(0.0 y \times 2.2 x$ & - & $(0.2 y \times 2.2 x)$ & - & - & - \\
\hline
\end{tabular}

The considered matrix is symmetric to the main diagonal that allows considering only one of its parts further on. Synthesis of hybrid structures of $H_{T E}$ class is possible only for objects of intrageneric level. As well as the genetically admissible variety of generating structures of $H_{T E}$ class within any geometrical genus is limited to 7 structural combinations, their total number within 6 genus will be presented by 42 dihybrid chromosomes (16).

$$
\sum H_{T E}=(C L ; K N ; P L ; T P ; S P ; T C) \times\left(\begin{array}{c}
(0.0 x \times 0.2 y) \\
(0.0 x \times 2.2 y) \\
(0.0 y \times 2.0 x) \\
(0.0 y \times 2.2 x) \\
(2.0 x \times 2.2 y) \\
(0.2 y \times 2.2 x) \\
(2.0 x \times 0.2 y)
\end{array}\right)
$$

The initial set of generating structures of dihybrids of the spatially-topological type $H_{G T}$ is defined by the mechanisms of intergeneric crossings, on condition of belonging of parental chromosomes to symmetry groups 0.0 and 2.2. The matrix of admissible crossings for each group is defined by 30 pair chromosomes of dihybrid type, Equation (16), and their total number (in the case of $m=2$ ) will be $\sum H_{G T}=30 \cdot 2=60$.

$$
\sum H_{G T}=(0.0 ; 2.2) \times\left(\begin{array}{l}
\left(C L_{x} \times C N_{y}\right) ;\left(C N_{x} \times C L_{y}\right) ;\left(P L_{x} \times C L_{y}\right) ; \\
\left(C L_{x} \times P L_{y}\right) ;\left(C N_{x} \times P L_{y}\right) ;\left(P L_{x} \times C N_{y}\right) ; \\
\left(C L_{x} \times T P_{y}\right) ;\left(C N_{x} \times T P_{y}\right) ;\left(P L_{x} \times T P_{y}\right) ; \\
\left(C L_{x} \times S P_{y}\right) ;\left(C N_{x} \times S P_{y}\right) ;\left(P L_{x} \times S P_{y}\right) ; \\
\left(C L_{x} \times T C_{y}\right) ;\left(C N_{x} \times T C_{y}\right) ;\left(P L_{x} \times T C_{y}\right) ; \\
\left(T P_{x} \times C L_{y}\right) ;\left(S P_{x} \times C L_{y}\right) ;\left(T C_{x} \times C L_{y}\right) ; \\
\left(T P_{x} \times C N_{y}\right) ;\left(S P_{x} \times C N_{y}\right) ;\left(T C_{x} \times C N_{y}\right) ; \\
\left(T P_{x} \times P L_{y}\right) ;\left(S P_{x} \times P L_{y}\right) ;\left(T C_{x} \times P L_{y}\right) ; \\
\left(T P_{x} \times S P_{y}\right) ;\left(S P_{x} \times T P_{y}\right) ;\left(T C_{x} \times T P_{y}\right) ; \\
\left(T P_{x} \times T C_{y}\right) ;\left(S P_{x} \times T C_{y}\right) ;\left(T C_{x} \times T C_{y}\right) ;
\end{array}\right)
$$

The element basis of spatially-electromagnetic chromosomes $H_{G E}$ is defined by the structure of 6 subgroups $(0.0 y ; 0.0 x ; 0.2 y ; 2.0 x ; 2.2 y$ and $2.2 x)$ for 6 geometrical genus $(C L, K N, P L, T P, S P, T C)$ considering restrictions for saving the electromagnetic symmetry of crossed chromosomes $\left(a_{2}=b_{2}=0\right)$. Therefore, synthesis of such structures is allowed only for the homologous chromosomes belonging to different genus. The quantitative structure of chromosomes within arbitrary genus is defined by 6 sets -3 for each direction of an electromagnetic 
field wave: $0.0 x ; 2.0 x ; 2.2 x$ - for $x$-oriented and $0.0 y ; 0.2 y ; 2.2 y$ - for $y$-oriented magnetic field sources. Each chromosomal set corresponds to 30 genetically admissible chromosomal combinations:

$$
N_{G E}=2 \cdot C_{6}^{2}=2 \cdot 6 \cdot 5 \cdot 4 \cdot 3 \cdot 2 / 2(6-2) \cdot 3 \cdot 2=30
$$

The total number of spatially-electromagnetic chromosomes within the first big period of GC is: $\sum N_{G E}=6 \cdot 30=$ 180 , and their variety is presented by a symmetrical matrix (Table 4).

Table 4. Matrix of admissible crossings of dihybrid structures of spatially-electromagnetic type (fragment)

\begin{tabular}{|c|c|c|c|c|c|}
\hline \multirow{2}{*}{$\begin{array}{l}\text { Components of genet } \\
\text { information of } \\
\text { chromosome } \boldsymbol{B}\end{array}$} & \multicolumn{5}{|c|}{ Components of genetic information of chromosome $\boldsymbol{A}$} \\
\hline & CLO.0x & KNO.0x & PLO.0x & TP0.0x & $\ldots$ \\
\hline$C L 2.0 x$ & - & $(K N 0.0 \times C L 2.0)$ & $(P L 0.0 \times C L 2.0)$ & $(T P 0.0 \times C L 2.0)$ & . \\
\hline$K N 2.0 x$ & $(C L 0.0 \times K N 2.0)$ & - & $(P L 0.0 \times K N 2.0)$ & $(T P 0.0 \times K N 2.0)$ & 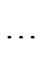 \\
\hline$P L 2.0 x$ & $(C L 0.0 \times P L 2.0)$ & $(K N 0.0 \times P L 2.0)$ & - & $(T P 0.0 \times P L 2.0)$ & $\cdots$ \\
\hline$T P 2.0 x$ & $(C L 0.0 \times T P 2.0)$ & $(K N 0.0 \times T P 2.0)$ & $(P L 0.0 \times T P 2.0)$ & - & .. \\
\hline$S P 2.0 x$ & $(C L 0.0 \times S P 2.0)$ & $(K N 0.0 \times S P 2.0)$ & $(P L 0.0 \times S P 2.0)$ & $(T P 0.0 \times S P 2.0)$ & \\
\hline$T C 2.0 x$ & $(C L 0.0 \times T C 2.0)$ & $(K N 0.0 \times T C 2.0)$ & $(P L 0.0 \times T C 2.0)$ & $(T P 0.0 \times T C 2.0)$ & .. \\
\hline$\cdots$ & $\ldots$ & $\ldots$ & $\ldots$ & $\ldots$ & \\
\hline
\end{tabular}

Similar matrixes represent genetically admissible variety of dihybrids for other 5 generating chromosomal combinations of the class $\sum H_{G E}$. It is easy to observe that the main diagonal of a matrix (Table 4) contains information on a subset of monohybrid structures of class $\sum H_{E}$, Equation (14), in a latent form.

Polyhybrid EM-objects $H_{G T E}$ form a class of the remote hybrids which synthesis is genetically admissible only at intergeneric level provided that crossed chromosomes are not homologs, i.e., don't belong to one group of symmetry $\left(a_{2} \neq b_{2}\right)$ and don't have the same orientability $\left(a_{3} \neq b_{3}\right)$. Taking into account the specified restrictions, the structure of polyhybrid classes can be received by association of subsets of dihybrid EM-structures of $H_{T E}$ class and monohybrid EM-structures of $H_{G}$ class.

As a result, the quantitative structure of generating chromosomes of the subprogram $H_{\text {GTE }}$ will be defined by the polyhybrid chromosomes allowing combination in spatial, topological and electromagnetic features:

$$
N_{\text {GTE }}=7 \cdot N_{G}=7 \cdot 30=210
$$

A fragment of a genetic databank of generating polyhybrid EM-structures $\left(\sum H_{G T E}\right)$ is given in Table 5 .

Table 5. Matrix of admissible crossings of polyhybrid structures of the class $\sum H_{G T E}$ (fragment)

\begin{tabular}{cccccc}
\hline Components of & \multicolumn{5}{c}{ Components of genetic information of chromosome $\boldsymbol{A}$} \\
\cline { 2 - 6 } $\begin{array}{c}\text { genetic information } \\
\text { of chromosome } \boldsymbol{B}\end{array}$ & $C L 0.0 x$ & $K N 0.0 x$ & $P L 0.0 x$ & $T P 0.0 x$ & $\ldots$ \\
\hline$C L 0.2 y$ & - & $K N 0.0 x \times C L 0.2 y$ & $P L 0.0 x \times C L 0.2 y$ & $T P 0.0 x \times C L 0.2 y$ & $\ldots$ \\
$K N 0.2 y$ & $C L 0.0 x \times K N 0.2 y$ & - & $P L 0.0 x \times K N 0.2 y$ & $T P 0.0 x \times K N 0.2 y$ & $\ldots$ \\
$P L 0.2 y$ & $C L 0.0 x \times P L 0.2 y$ & $K N 0.0 x \times P L 0.2 \mathrm{y}$ & - & $T P 0.0 x \times P L 0.2 y$ & $\ldots$ \\
$T P 0.2 y$ & $C L 0.0 x \times T P 0.2 y$ & $K N 0.0 x \times T P 0.2 y$ & $P L 0.0 x \times T P 0.2 y$ & - & $\ldots$ \\
$S P 0.2 y$ & $C L 0.0 x \times S P 0.2 y$ & $K N 0.0 x \times S P 0.2 y$ & $P L 0.0 x \times S P 0.2 y$ & $T P 0.0 x \times S P 0.2 y$ & $\ldots$ \\
$T C 0.2 y$ & $C L 0.0 x \times T C 0.2 y$ & $K N 0.0 x \times T C 0.2 y$ & $P L 0.0 x \times T C 0.2 y$ & $T P 0.0 x \times T C 0.2 y$ & $\ldots$ \\
$\ldots$ & $\ldots$ & $\ldots$ & $\ldots$ & $\ldots$ & $\ldots$
\end{tabular}

The main diagonal of a matrix (Table 5) contains information on generating dihybrid structures of $\sum H_{T E}$ class, Equation (16), in a latent form. 
The results of analysis of crossing space, by criterion of hereditary information components, can be presented in the form of the generalized genetic program which hierarchical structure includes 7 local subprogrammes (Figure 6).

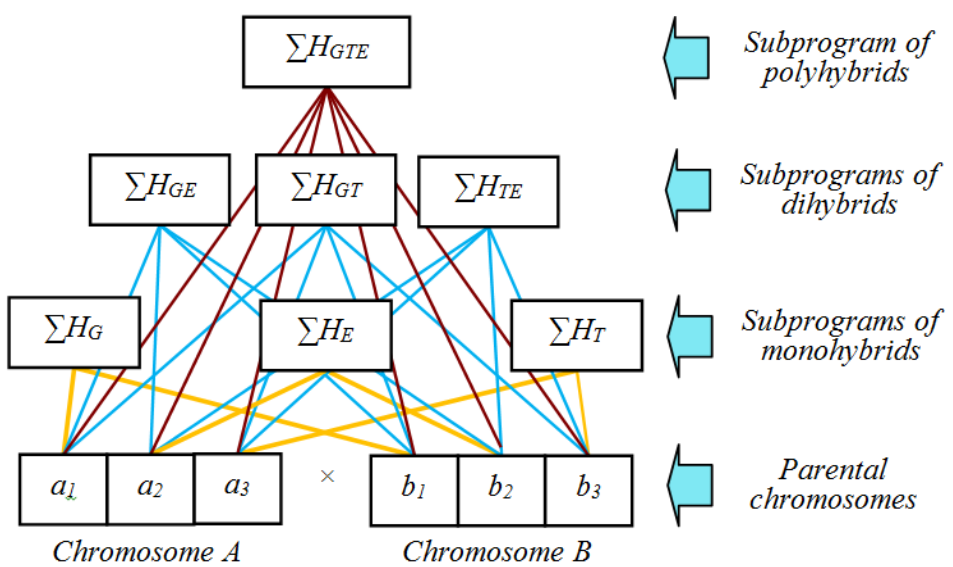

Figure 6. Architecture of the generalized genetic program of hybrid classes of EM-objects on the basis of inherited information

The genetic hybridization program (Figure 6) is invariant to the evolution time, level of structural complexity and area of functioning of descendant structures, i.e., represents the generalized architecture which, in essence, is a form of presentation of the genetic law (taking into account the accepted assumptions) of the structural organization of hybrid EM-objects. The program contains information on quantitative structure and hereditary features (spatial, topological and electromagnetic) of hybrid Species and functional classes of EM-objects taking place and appearing in the process of technical evolution. The quantitative analysis results of generating chromosomes and an admissible variety of Species of hybrid EM-objects are generalized in Table 6.

Table 6. Quantitative structure of genetically admissible variety of hybrid Species of EM-objects

\begin{tabular}{lccccccc}
\hline \multirow{2}{*}{ Species of hybrid EM-objects } & \multicolumn{7}{c}{ Sets of generating chromosomes } \\
\cline { 2 - 7 } & $H_{G}$ & $H_{E}$ & $H_{T}$ & $H_{G T}$ & $H_{T E}$ & $H_{G E}$ & $H_{G T E}$ \\
\hline $\begin{array}{l}\text { Number of generating chromosomes in } \\
\text { the genetic program }\end{array}$ & $\begin{array}{c}15 \\
(m=6)\end{array}$ & $\begin{array}{c}6 \\
(m=6)\end{array}$ & $\begin{array}{c}2 \\
(m=6)\end{array}$ & $\begin{array}{c}30 \\
(m=2)\end{array}$ & $\begin{array}{c}7 \\
(m=6)\end{array}$ & $\begin{array}{c}30 \\
(m=6)\end{array}$ & $\begin{array}{c}30 \\
(m=7)\end{array}$ \\
\hline $\begin{array}{l}\text { Genetically admissible variety of hybrid } \\
\text { descendant Species }\end{array}$ & 90 & 36 & 12 & 60 & 42 & 180 & 210 \\
\hline
\end{tabular}

\section{Evolutionary Experiment}

Reliability of genetic hybridization models and genetic programs was defined by evolutionary experimental set up. Evolutionary experiments in structural Electromechanics represent the new methodology of researches focused on verification of genetic evolution theory provisions (Shinkarenko \& Kotlyarova, 2012; Shinkarenko et al., 2013). The main idea of evolutionary experiments lies in establishment of degree of a structural and information correspondence between genetic programs and a real variety of EM-objects involved in technical evolution.

In evolution process $\left(T_{E} \neq 0\right)$ the genetically admissible variety of Species of hybrid EM-objects $S_{H}$ ordered by their genetic program $P_{G}$, can be presented as the sum of two subsets

$$
S_{H}=\left(S_{R}+S_{F}\right) \subset P_{G},
$$

Where $S_{R}$ are the structural representatives of real and informational Species, which have appeared during the evolution period $T_{E} ; S_{F}$ is a variety of Species of implicit type, i.e., genetically admissible, but still absent for this time of evolution. 
It is quite obvious that with increase of $T_{E}$ the ratio between subsets of $S_{R}$ and $S_{F}$ will change towards increase in multiplicity of $S_{R}$, on the assumption that the multiplicity of $S_{H}$ is constant. Thus, proceeding from the structure of the set $S_{H}$, it is possible to separate two approaches to the organization and carrying out the evolutionary experiments: genomic-historical and genomic-predictive.

Practical implementation of the unique program of genomic-historical experiments is realized in NTUU "KPI", with the assistance of research groups of other technical universities, since 1998. Experiment is carried out for genetic programs of separate functional classes, as well as for single EM-objects. The results of patent and information search with the subsequent genomic analysis of known structural representatives (real and informational) created in the process of their technical evolution are used as a source of information about the structural variety of EM-objects.

Comparison of results of the genomic analysis of genetic programs with representatives of hybrid EM-objects of real evolution, was carried out on several functional classes at the same time: electromagnetic separators (Shvedchikova, 2013), electrical motors with the rolling rotor (Shinkarenko, Naniy, Kotlyarova, \& Dunev, 2012), combined electromechanical systems of "motor-wheel"-type (Shinkarenko \& Avgustinovich, 2007) and "spindle-motor"-type) (Shinkarenko et al., 2013), etc. The results of conducted experiments confirmed reliability of the hybridization principles and genetic models of the structural organization and evolution of hybrid EM-objects.

The purpose of genomic and predictive experiments lies in experimental confirmation of predictive function of the genetic programs. The program of such experiments represents time-research process providing the next:

- Definition and interpretation of genetic programs, analysis of Species of the hybrid structures which are absent for this time of evolution;

- $\quad$ Directed synthesis and innovative selection of EM-structures;

- Development of technical solutions;

- Documental or technical confirmation of novelty and competitiveness of the created objects.

Thus, the experiment is directed on checking of reliability of results of genetic anticipation and methodology of the directed synthesis of new EM-objects according to their genetic programs (genetic codes). There were no analogical scientific problems in technical science stated in such way.

Practical realization of predictive experiment, in essence, represents the scenario of operated evolution realized by the use of genetic program prognostic potential of a studied class of EM-objects. Documentarily confirmed fact of input in evolution of new competitive technical solutions (Figures 3 - 5) which, in essence, are technical objects of the hybrid and combined types being the first-ever synthesized by the genetic codes (Shinkarenko et al., 2010, 2012, 2013), became the end result of evolutionary experiment.

\section{Conclusion}

It is for the first time shown that hybrid objects represent an independent and predictable branch in structural evolution of the complex electromechanical systems, covering all generic categories, irrespective to their functional accessory. Structural evolution of hybrid EM-objects is predetermined by their genetic programs representing final sets of hybrid electromagnetic chromosomes.

The generalized genetic program of hybrid EM-objects which represents the hierarchical structure consisting of 7 subprograms is for the first time defined and decoded. Local subprograms contain information on genetically admissible variety of descendant objects (both existing, and potentially possible) by criterion of crossed genetic information.

By the results of programs decoding the quantitative information on genetically admissible variety of hybrid Species of EM-objects is received and their genetic classification by inherited properties is carried out. System interrelations between the genetic information of crossed chromosomes and periodic structure of generating system of primary sources of a field, and also electromagnetic, topological and geometrical properties of descendant structures are scientifically proved, confirming fundamentality and consistency of the hybrid EM-objects theory provisions.

The research results represent further development of the theory of hybrid EM-structures and form a system basis for creation of informational and genetic databanks and innovative knowledge bases, genetic systematics development, and also statements of problems of the hybrid EM-objects directed synthesis along the set criterion function. 


\section{References}

Breedveld, P. (2001). A generic dynamic model of multiphase electromechanical transduc- tion in rotating machinery, Proceedings WESIC 2001, June 27-29, 2001, University of Twente, Enschede, The Netherlands (pp. 381-394).

Chiasson, J. (2005). Modeling and High Performance Control of Electric Machines. John Wiley \& Sons Inc. http://dx.doi.org/10.1002/0471722359

Kalachek, P. M., Koriagin, S. I., Kolesnikov, A. V., \& Minkova, E. S. (2011). Hybrid Adaptive Intelligence Systems. Part 1: Theory and technology of development: Monograph. Kaliningrad: I. Kant BFU.

Kovalchuk, M. B. (2011). Convergence of sciences and technologies - break in the future. Russian technologies. No. 1 - 2. (pp. 13-23).

Kuznietsov, Y. N., \& Dmitriev, D. A. (2009). Concept of Hybrid Machine Configuration With Parallel Kinematics Based on Modular Principle. Materials of Int. Sci. Conf. "Thechnic, Technology and Systems “Tekhsis 2009”. Plovdiv: Technical University Sofia. (pp. 19-36).

Rodriguez, H., \& Ortega, R. (2003). Stabilization of electromechanical systems via inter- connection and damping assignment. Int. Journal of Robust and Nonlinear Control, 13, 1095-1111. http://dx.doi.org/10.1002/rnc.804

Shinkarenko, V. F. (2002). Bases of Electromechanical Systems' Evolution Theory. Kyiv, K.: Naukova dumka.

Shinkarenko, V. F. (2013). Genetic programs of structural evolution of anthropogenic systems. (Interdisciplinary aspect). Works of Tavriysky state agrotechnical university, 13(4), 11-20.

Shinkarenko, V. F., \& Avgustinovich, A. A. (2007). Genome Structure and Macroevolutional Analysis of Combined Electromechanical Systems of "Motor-mover"-type. Visnyk of Mykhailo Ostrogradskyi KDU, $3(2), 22-26$.

Shinkarenko, V. F., Chumack, V. V., Gaidaienko, Iu. V., \& Miroshnyk, O. L. (2010). Electromechanical System for Steel Pipe Transportation. Patent of Ukraine, No. 97186, Int. Cl. H02K 41/025, B65G 23/22. Application No. a 2010 07687, 18.06.2010. Published from 10.01.2012, 1.

Shinkarenko, V. F., Chumack, V. V., Gaidaienko, Iu. V., \& Vyshnevskyy, T. S. (2012). Brushless Synchronous Generator. Patent of Ukraine, No. 77370, Int. Cl. H 02K 21/14. Application No. u 2012 09511, 06.08.2012. Published from 11.02.2013, 3.

Shinkarenko, V. F., \& Gaidaienko, Iu. V. (2010a). Genetic Structurization Principles of Hybrid Electromechanical Structures. Visnyk of Mykhailo Ostrogradskyi KDU, 3(2), 47-50.

Shinkarenko, V. F., \& Gaidaienko, Iu. V. (2010b). Structural and System Analysis of Hybrid Electromechanical Objects at Intrageneric Level. Electrical Engineering and Electromechanics, 5, 30-33.

Shinkarenko, V. F., Gaidaienko, Iu. V., \& Miroshnik, O. L. (2010). Structural foresight and streamlined synthesis models of hybrid electromechanical objects at intrageneric level. Collection of scientific works «Electrical machines theory and design issues». Ulianovsk: UlGTU, 105-112.

Shinkarenko, V. F., Gaidaienko, Iu. V., \& Ahmad, N. Al. H. (2013). Genetic Programs of Structural Evolution of Hybrid Electromechanical Objects. International journal of Engineering \& Technology, 1(2), 44-49.

Shinkarenko, V. F., \& Kotlyarova, V. V. (2012). Theory and Practice of Operated Evolution at the Level of Certain Species of Electromechanical Energy Converters. Proceedings of the Tavria State Agrotechnical University, 2(1), 3-14.

Shinkarenko, V. F., Kuznietsov, Y. N., Gaidaienko, Iu. V., Kovtun, A. S., \& Oliynyk, K. O. (2013). Spindle Unit for Machine-tool. Patent of Ukraine, No. 82880, Int. Cl. B23B 19/00, B23B 47/00. Application No. u 2012 11168, 26.09.2012. Published from 27.08.2013, 16.

Shinkarenko, V. F., Kuznietsov, Y., Gaidaienko Iu. V., \& Oliynyk, K. (2013). The Operability Analysis of Spindle-Motors Hybrid Electromechanical Systems. 13th Anniversary International scientific Conference «Unitech'13». Gabrovo, Bulgaria. III, 268 - 272.

Shinkarenko, V. F., Naniy, V. V., Kotlyarova, V. V., \& Dunev, A. A. (2012). Genetic Program of Structural Evolution of Electrical Motors With Rolling Rotor. Electrical Engineering and Electromechanics, 2, 42.

Shinkarenko, V. F., Shvedchikova, I. A., \& Kotlyarova, V. V. (2013). Evolutionary Experiments in Genetic Electromechanics. 13th Anniversary International scientific Conference «Unitech'13». Gabrovo, Bulgaria, 
III, 289-294.

Shvedchikova, I. A. (2013). Evolutionary-experimental Research of Functional Class of Magnetic Separators. Proceedings of the Tavria State Agrotechnical University, 13(4), 96-103.

Sonin, A. S. (2006). Course in Macroscopic Crystal Chemistry. Moscow, M.: Phizmatlit.

Tadmor, G. (2002). On approximate phasor models in dissipative bilinear systems. IEEE Trans. on Circuits and Systems I, 49, 1167-1179. http://dx.doi.org/10.1109/TCSI.2002.801251

Venda, V. F. (1990). Hybrid Intelligence Systems: Evolution, Psychology, Informatics. Moscow, M: Mashinostroenie.

Wang, Y., Stefanopoulou, A., Haghgooie, M., Kolmanovsky, I., \& Hammoud, M. (2000). Modelling of an Electromechanical Valve Actuator for a Camless Engine. 5th International Symposium on Advanced Vehicle Control, Ann Arbor, Michigan USA.

Yerosh, I. L. (2001). Discrete mathematics. Combinatorics: Educational book. St. Petersburg, SPb.: GUAP.

\section{Copyrights}

Copyright for this article is retained by the author(s), with first publication rights granted to the journal.

This is an open-access article distributed under the terms and conditions of the Creative Commons Attribution license (http://creativecommons.org/licenses/by/3.0/). 